\title{
Análise de estruturas sujeitas a ações sísmicas utilizando o método dos elementos finitos
}

\section{Analysis of Structures Subject to Seismic Actions Using Finite Element Method}

\author{
Leandro F. Andrioli ${ }^{1}$, Antonio R. F. Neto ${ }^{1}$, Everton C. Medeiros ${ }^{1}$, Sofia Glyniadakis ${ }^{1}$,
}

Marcelo S. Martins*1

\begin{abstract}
RESUMO
Este estudo aborda a verificação estrutural de um equipamento que pode sofrer cargas sísmicas. A avaliação adequada das cargas é extremamente importante para a segurança e eficácia estrutural e, como no Brasil quase todas as estruturas não requerem consideração da ação sísmica em seus projetos, a aplicação dessas cargas é frequentemente negligenciada, mesmo quando a estrutura é para exportação. Este estudo visa ilustrar a criticidade desta questão. Para essa avaliação, uma ferramenta cada vez mais comum entre os engenheiros é usada, o Método dos Elementos Finitos. A especificação técnica ETG1015 é usada para a preparação desta avaliação, que estabelece quais cargas devem ser aplicadas a estruturas sujeitas a terremotos para o projeto El Quimbo. As cargas aplicadas à estrutura, de acordo com ETG1015, são acelerações laterais simulando condições sísmicas. As análises estruturais são feitas pelo Método dos Elementos Finitos, utilizando o software Cosmos. Desse modo, é possível determinar tensões na estrutura e, portanto, comparar com os valores permitidos pela norma AISC e, se necessário, modificações de projeto visando o cumprimento das normas propostas.
\end{abstract}

Palavras-chave: MEF, Elementos finitos, Análise estrutural, Cargas sísmicas

\section{ABSTRACT}

This study approaches the structural verification of a piece of equipment that may suffer seismic loads. The proper evaluation of loads is extremely important for safety and structural effectiveness and, as in Brazil almost all structures do not require consideration of seismic action in their designs, the application of these loads is frequently overlooked, even when the structure is for exportation. This study aims to illustrate the criticality of this issue. For this evaluation, an increasingly common tool among engineers is used, the Finite Element Method. The technical specification ETG1015 is used for the preparation of this evaluation, that establishes which loads should be applied to structures subjected to earthquakes for the project El Quimbo. The loads applied to the structure, according to ETG1015, are lateral accelerations simulating earthquake conditions. Structural analyses are made by the Finite Element Method, using Cosmos software. Thereby, it is possible to determine stresses on the structure and therefore compare with the allowable values by the AISC standard and, if necessary, design modifications targeting the fulfillment of standards proposed.

Keywords: FEM, Finite elements, Structural analysis, Seismic loads 


\section{INTRODUÇÃO}

Os terremotos, ou sismos, são fenômenos naturais, que mesmo apesar dos esforços despendidos para a clarificação destes fenômenos, eles continuam imprevisíveis no tempo e espaço, o que pode vir a ter consequências catastróficas, tanto economicas quanto sociais.

Segundo Buchholddt (1999), a crosta terrestre é dividida em placas tectônicas, as quais caracterizam-se por serem extensos conjuntos de rochas, que são limitadas por regiões de deslizamento. Como descrito por McGuire (1968), nas fronteiras entre elas, podem vir a ocorrer interações, as quais podem ser intensas ou mais amenas, essas interações geram ondas mecânicas propagadas através do solo, que são conhecidas como terremotos.

O objetivo principal deste trabalho é comprovar a possibilidade de utilização do método de elementos finitos para a avaliação de estruturas sujeitas a ações sísmicas utilizando o método da análise estática equivalente, em uma estrutura de base que suporta outro conjunto, do qual não se tem informações detalhadas.

Esse trabalho também visa demonstrar a importância da consideração das cargas provenientes de sismos em estruturas a serem implantas em zonas sísmicas, fato muitas vezes esquecido no Brasil.

\section{METODOLOGIA}

Para a verificação estrutural do suporte do interruptor é necessário a utilização de normas que forneçam os carregamentos e método de aplicação, bem como tensões e esforços admissíveis. A seguir estão listadas as normas e os softwares utilizados, e uma breve descrição de sua finalidade:

- Software DraftSight: Utilizado na visualização dos desenhos 2D.

- Software Cosmos: Utilizado para a elaboração do modelo matemático (elementos finitos) e para a análise estrutural.

- Especificação técnica ETG 1015: Através desta norma são determinados os carregamentos considerados na análise estrutural do suporte.

- Norma AISC: A norma AISC é utilizada para a determinação das tensões admissíveis para a estrutura.

- Norma NBR 8800: Através desta norma são determinados os esforços admissíveis para os parafusos, olhais e soldas existentes na estrutura. 
Com essas premissas estabelecidas o projeto básico é feito, e com estas informações faz-se o modelo de elementos finitos, com o modelo finalizado são elaboradas as análises estruturais, de acordo com os passos a seguir:

$1^{\circ}$ ) Análise modal para determinação da frequencia natural de vibração;

$2^{\circ}$ ) Aplicação das cargas sísmicas e análise estática equivalente para verificação da estrutura;

$3^{\circ}$ ) Verificação dos elementos de ligação.

Após a obtenção dos resultados das análises usando dados da Tabela 1, as tensões encontradas são comparadas com as tensões admissíveis, caso as tensões encontradas não sejam menores que as admissíveis são efetuadas alterações de projeto ou material até se obter resultados satisfatórios.

Tabela 1 - Características da estrura.

\begin{tabular}{|l|l|}
\hline Massa (estrutura +interruptor) & $9715,0 \mathrm{~kg}$ \\
\hline Comprimento & $6576,0 \mathrm{~mm}$ \\
\hline Largura & $4327,0 \mathrm{~mm}$ \\
\hline Altura & $1179,0 \mathrm{~mm}$ \\
\hline
\end{tabular}

A estrutura (Figura 1) é toda construída em aço ASTM A36, os parafusos são todos M16 classe 8.8 e os chumbadores segundo ASTM A193 grau B7.

Figura 1 - Desenho do suporte.

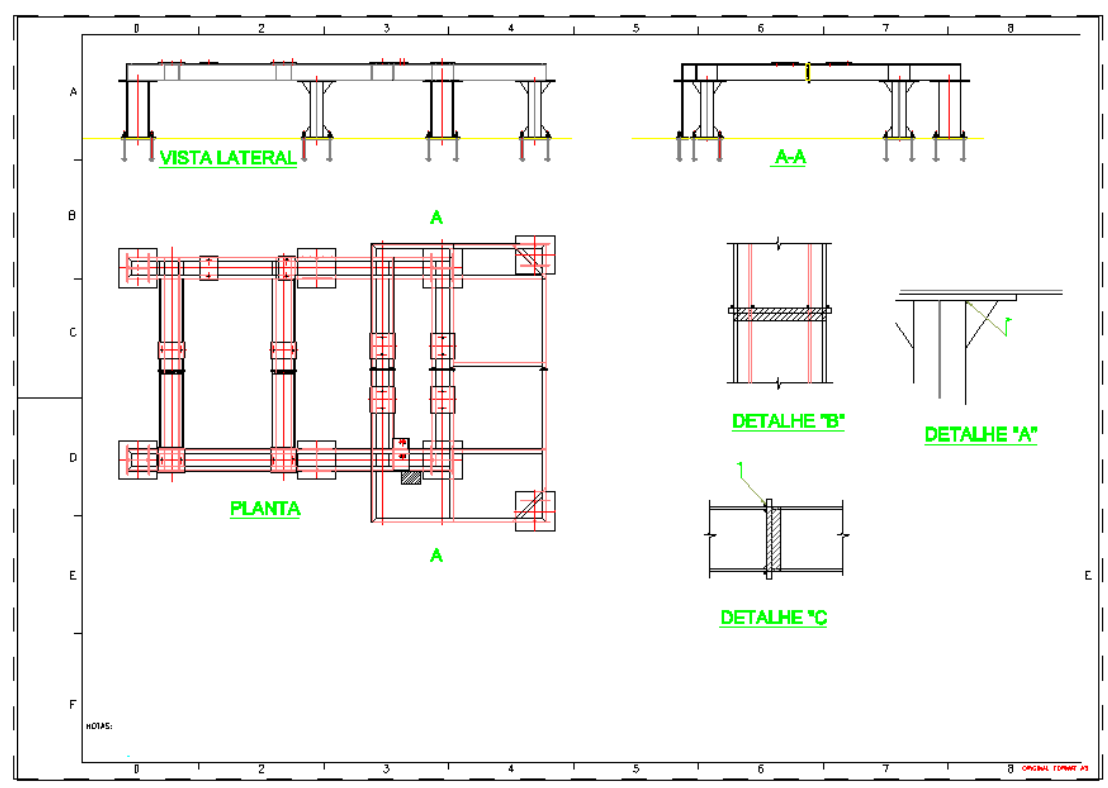


O modelo de elementos finitos é construído utilizando elementos de placa (SHELL4), elementos de viga (BEAM3D), elementos rígidos (RBAR), elementos de massa (MASS) e elementos de contato (GAP) (Figura 2).

Figura 2 - Detalhe dos componentes estruturais.

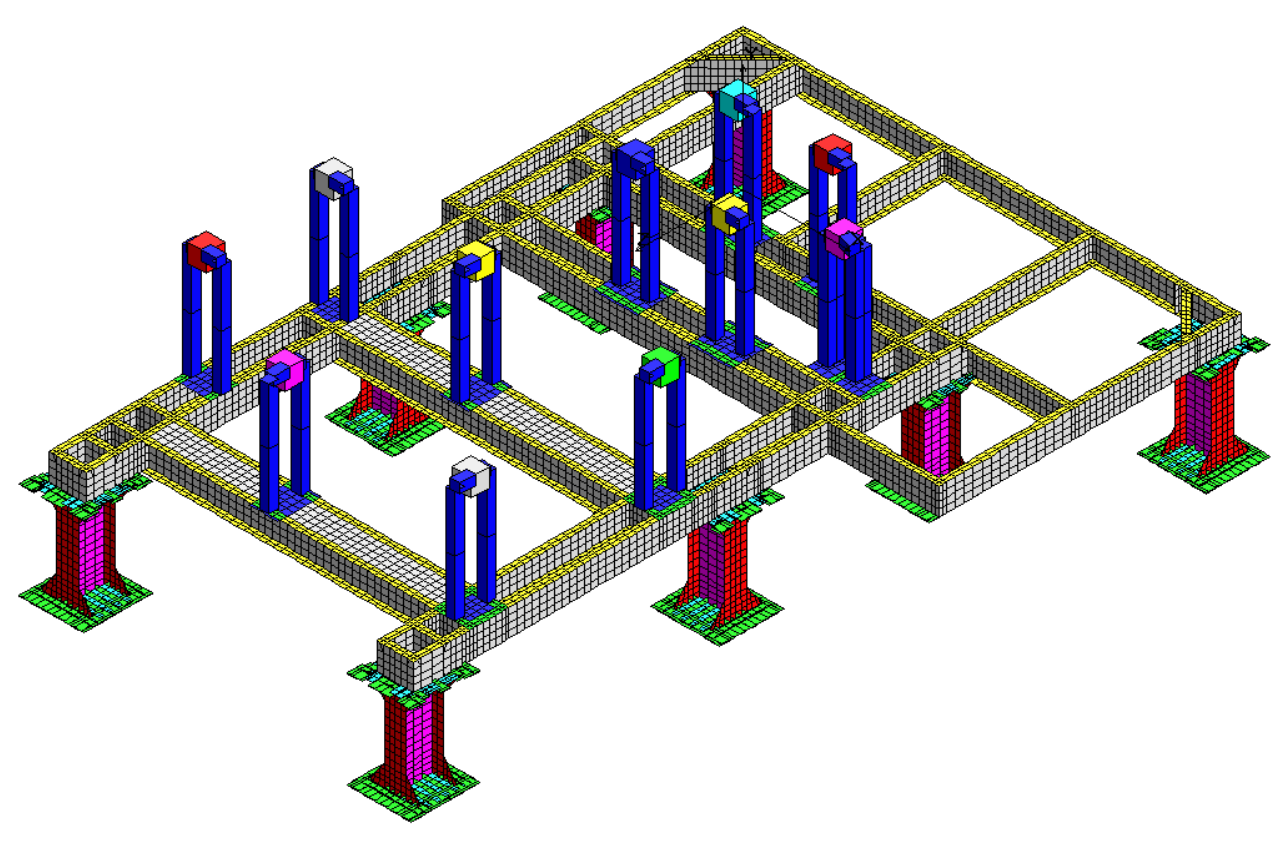

A condição de contorno utilizada é a restrição da translação no sentido x y, e z nos nós da base dos chumbadores. O contato da estrutura com a base é considerado por elementos de contato, na base dos elementos de contato são consideradas restrições translacionais e rotacionais. As reações de apoio encontradas nos nós dos chumbadores são utilizadas para a verificação dos chumbadores. As cargas são impostas de acordo com a ETG-1015, seção 02.07.06.

Considerando o pico do gráfico de resposta espectral de vibrações, referente a $2 \%$ de amortecimento obtem-se o valor de de 1,37 para a aceleração espectral. De acordo com a seção 01.04 se os equipamentos das categorias B e C pode-se multiplicar as ordenadas por 0,8 e 0,6 respectivamente, assim sendo o pico para o amortecimento de $2 \%$ para a estrutura a ser analisada é de $0,8 \times 1,37$, que resulta numa ordenada máxima de 1,096 .

\section{RESULTADOS E DISCUSSÃO}

Os resultados para o caso de carga que apresenta menor margem de segurança, o caso de carga LC3, o qual considera o peso próprio, carga sísmica vertical no sentido 
negativo do eixo $\mathrm{Y}$, carga sísmica horizontal no sentido oblíquo dos eixos $\mathrm{X}$ e $\mathrm{Z}$ e a carga de curto-circuito podem ser visualizados na Figura 3 a seguir.

\section{Figura 3 - Tensões LC3 (Von Mises) [Pa].}

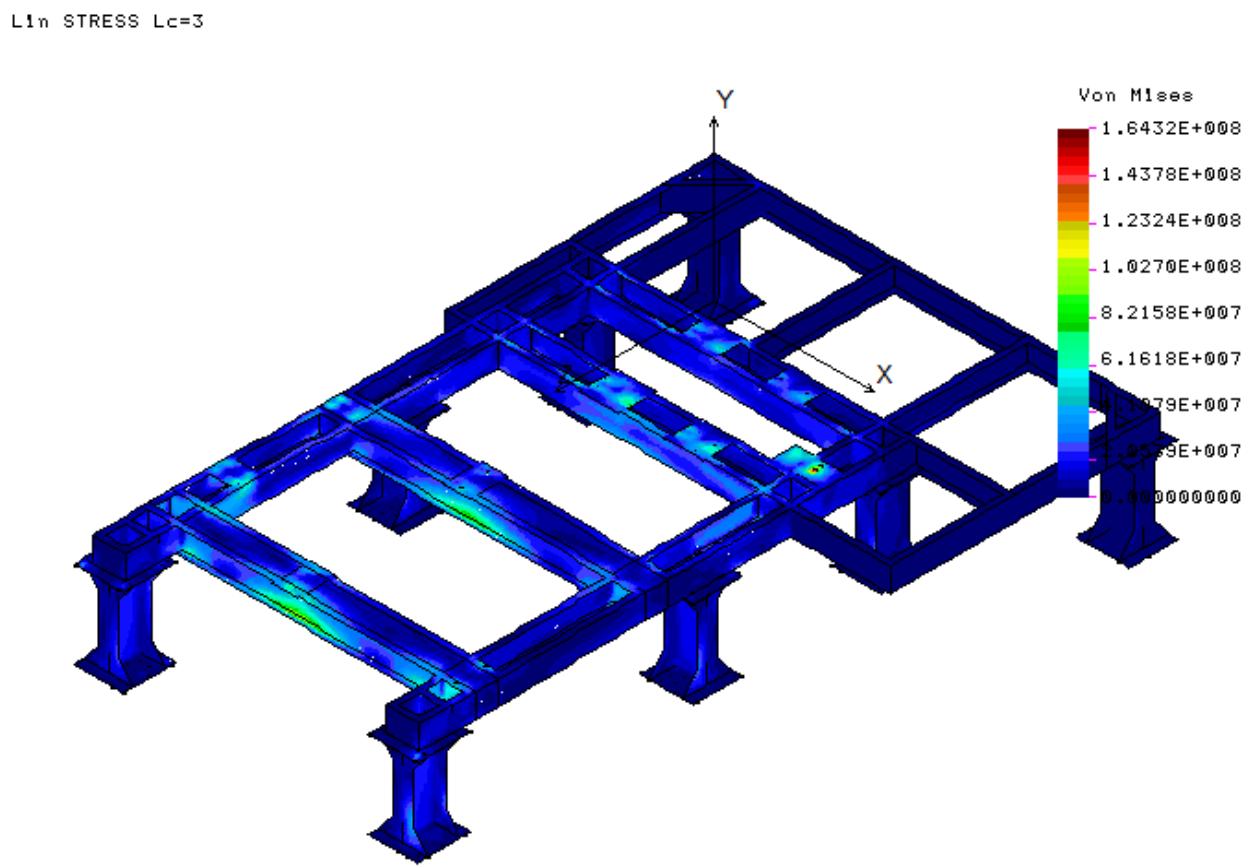

\section{CONCLUSÃO}

As tensões atuantes e esforços máximos resultantes das análises e verificações são inferiores aos admissíveis, desta forma, não são esperados problemas estruturais no suporte do interruptor, sujeito a cargas de curto circuito e cargas sísmicas.

Além de se avaliar a estutura do suporte do interruptor, o trabalho também demonstrou a metodologia de cálculo para estruturas sujeitas a cargas sísmicas, assim, podemos expandir o raciocínio adotado neste trabalho para outras estruturas, atentando sempre para as curvas de espectro de resposta, que dependem da frequência natural da estrutura e amortecimento modal.

\section{REFERÊNCIAS}

AMERICAN INSTITUTE OF STEEL CONSTRUCTION. AISC 360-05: Specification for structural steel buildings. Chicago, 2005. 
ASSAN, A. E. Método dos Elementos Finitos. $2^{\text {a }}$ edição. Campinas: Editora da Unicamp, 2003.

ASSOCIAÇÃO BRASILEIRA DE NORMAS TÉCNICAS. NBR 8800: Projetos de estruturas de aço e de estruturas e concreto de edifícios. Rio de Janeiro, 2008.

AZEVEDO, A. F. M. Mecânica dos Sólidos e Métodos dos Elementos Finitos. Faculdade de Engenharia da Universidade do Porto, 1996.

AZEVEDO, A. F. M. Método dos Elementos Finitos. Porto: Faculdade de Engenharia da Universidade do Porto, 2003.

BUCHHOLDT, H. A. An introduction to cable roof structures. 2nd ed. London: Thomas Telford,1999. 285 p.

COMISIÓN FEDERAL DE ELECTRICIDAD (CFE). Manual de diseño de obras civiles: Estructuras: criterios de diseño: C.1.3. Diseño por sismo. México, DF, 1993. $36 \mathrm{p}$.

ESPECIFICACIONES TECNICAS GENERALES, DISEÑO SISMICO: ETG1015, ENDESA, 1987.

GESUALDO, F. A. R. Método dos Elementos Finitos. 2010. 53 f. Monografia (Especialização) - Curso de Pós-graduação em Engenharia Cívil, Universidade Federal de Uberlândia, Uberlândia, 2010.

GUIMARÃES, W. R. S. Método dos Elementos Finitos Generalizados com Continuidade C1 Aplicados a Placas de Kirchhoff. 2006. 140 f. Dissertação (Mestrado) - Curso de Pós Graduação em Engenharia Mecânica, Puc - Mg, Belo Horizonte, 2006.

McGUIRE, W. Steel structures. Englewood Cliffs: Prentice-Hall, 1968, 1112 p.

MIRANDA, D. H. L. Análise estrutural do suporte de pastilha de grafite para lubrificação de roda ferroviária. Guaratinguetá, 2015. 89 p. Monografia (Graduação em Engenharia Mecânica) - Faculdade de Engenharia, Campus de Guaratinguetá, Universidade Estadual Paulista. 
MOREIRA, A. F. R. Caracterização do fator de intensidade de tensão em corpos trincados através do método dos elementos finitos. Guaratinguetá, 2015. 68 p. Monografia (Graduação em Engenharia Mecânica) - Faculdade de Engenharia, Campus de Guaratinguetá, Universidade Estadual Paulista.

TAVARES, A. G. S. Método dos Elementos Finitos. 2005. Dissertação (Mestrado) - Curso de Mecânica Espacial e Controle, Instituto Nacional de Pesquisas Espaciais, São José dos Campos, 2005.

Recebido em: 01/09/2021

Aprovado em: 25/09/2021

Publicado em: 30/09/2021 